\title{
On Exploration of Various Success Factors and Challenges for Handloom and Carpet Weaving Textile Sector in India
}

\author{
Yogender Singh \\ Ex- PhD Research Scholar \\ Department of Operational \\ Research, University of Delhi, \\ Delhi, India
}

\author{
Remica Aggarwal \\ MIT-SOER, MIT- ADT University \\ Pune, India
}

\author{
Viraj Voditel \\ Techture Structures Private \\ Limited \\ Nagpur, India
}

\begin{abstract}
Following research paper discusses the various success factors and challenges for carpet weaving industries in India in improving the status of the same. Further, the interrelationships have been studied with the help of ISM methodology . Being a deeply traditional industry, carpet making has immensely influenced its production process and value chain. In this paper, the authors discuss the application of technological elements to the hand woven carpet industry, indicating that traditional technologies can be renewed by the use of the latest innovative advantages.
\end{abstract}

\section{Keywords}

Carpet weaving ; ISM methodology ; handloom and weaving industry

\section{INTRODUCTION}

The art of weaving is perhaps as old as human civilization. Carpet weaving constitute a timeless facet of the rich cultural heritage of Asian countries. The carpet industry is one of the most rapidly growing traditional industries in India. Carpet industry is a subset of handicraft and cottage industry and provides jobs right at doorstep of more than 100,000 workers, belonging to economically backward class of society. Jaipur and its adjacent districts are the major manufacturing clusters in Rajasthan that shares a substantial part $(15 \%)$ of total carpet manufacturing from India [1-3]. The Indian hand-made carpet industry is an export-oriented industry and is a significant contributor to the country's rural economy, which helps in providing employment. India has made remarkable progress in export market of hand knotted carpets as well. The production of hand knotted carpets is very labour intensive and time consuming. At international level, Iran, China, India and Nepal are the key players in the global carpet trade. Other significant exporters include Turkey, Afghanistan, and Pakistan. America and Europe are the chief importers.

\subsection{Traditional Carpet manufacturing process}

Traditionally, all the designs that were used in the carpet were made manually. Design outline is prepared on white paper and then filled with appropriate colours to make them different from existing designs. The same design was used to be converted into different graph papers to enable weavers understand them clearly so that while interlocking of wool into cotton they could interpret the designs accurately. After the finalization of design a reduced graphical format of the complete design was prepared and given to weavers. The whole designing process used to take around 20-25 days depending on the compactness and size of the carpet [4-5].

\section{LITERATURE REVIEW}

This section is divided in to three parts. Section 2.1 deals with the history of carpet industry. Section 2.2 and section 2.3 describes the success factors and the challenges faced by traditional carpet and handloom manufacturing industries in India.

\subsection{History of carpet industry}

The carpet industry is a de-centralized industry [6] and represents the craft which is carried forward from generations to generations. Egypt is recognized as the original home of carpet making. The manufacture of carpets was later taken up at Alexandria, and from Alexandria it was taken into Western India [7]. The Mughal king Akbar is credited with the introduction of pile carpets into India, but Jahangir and Shahjahan brought the art of Indian carpets to its prominence [8-10]. Many of the carpets produced during the reign of Shahjahan are housed in the Victoria and Albert museum in London and in other museum across the Europe, including a few in the Jaipur museum in India [9]. In the post-British period, carpet making was taken up by many industries which flourished in the regions located in Bhadohi, Mirzapur, and Agra in the state of Uttar Pradesh, Jaipur in the state of Rajasthan and in the Kashmir valley. The MirzapurBhadohi region is also known as "carpet- belt" and accounts for about 85 per cent of Indian carpet exports.

\subsection{Challenges behind the handloom and carpet weaving [11-14]}

According to Singh [11] many Indian handicrafts succumbed to the challenges of technological modernisation, the carpet industry, one of the urban handicrafts has not only successfully withstood the onslaught of technological development but has flourished over the years. This is due to the adaptability that the industry has shown to new situations. Venkateswarlu et al. [12] argued that there was a significant decline in the production and exports of hand knotted carpets particularly in the core carpet belt of Mirzapur-Bhadohi. The recent slump has affected all the sections involved in this business. The prospect of carpet industry is very much dependent on the exports. Export prospects of carpets basically depend on overseas demand and domestic supply potential Tyagi [13]. Pandey[14] analyzed the impact of recession on carpet industry.

Following are the major challenges or barriers observed :

2.2.1Monotonous designs (MD) : The manual design process had its own limitations and therefore the market got saturated with similar designs. Designers could modify color combinations, but market 
acceptability started dwindling. The designs could not be easily renewed. There was a need to introduce new range of designs to compete in the global market.

2.2.2Expensive methods of designing (EM) : Creation of designs with various color combinations is an important aspect of selling the product. But traditional methods are expensive when different colour combinations are to be used.

2.2.3 In appropriate calculation of raw material requirements (ICRM) : There are no accurate methods for calculation of the raw material requirements. To avoid the problem of shades they used to dye more wool than their requirement. Hence $10-12 \%$ of raw material was getting wasted which accounted for $3-5 \%$ of the total cost. The possibility of reusing and recycling these waste raw materials are limited, as shades could not be easily mixed.

2.2.4 Presence of manual errors (PME) : The designs prepared on the graph paper when transferred for weaving on the carpet varied because of manual errors, resulting in poor customer acceptance. In the case of high knotting density carpets, graph papers are of rather limited use.

2.2.5 Limited varieties (LV) : Varieties were limited and acceptance was low. Varieties were limited and acceptance was low. In the traditional carpet technology there was no way one could make a variety of high knotting density carpets.

2.2.6 Repetitions of designs (RoD) : There was no systematic method for varying designs, in the manual methods; hence designs were simply repeated. This also resulted in poor customer acceptance.

2.2.7 Storage and handling problems (SHP) : Storage and handling of the graph paper based designs had its own problems as they degraded quickly. As a result the design had to be repeated afresh on a new graph paper, which is time-consuming and expensive.

2.2.8 Violation of Intellectual property rights (VIPR) : The intellectual property rights of the manual designs were rarely respected as a result the money, time and effort spent in acquiring a new design was rarely yielding benefits. Piracy of designs was a major systemic problem.

2.2.9Time factor (TF) : Time factor is also one of the major problems as the entire process is hand-made and very cumbersome. Unavailability of the designs restricted the production when the sales did not move, weavers incurred heavy losses due to accumulated stocks and the weavers resorted to debts to reduce losses.

Besides the above, some of the obvious challenges are as follows:

2.2.10 Infrastructural constraints (IC) :

2.2.11 Low use of technologies (LUT) :

2.2.12 Low wages to workers (LWW):
2.2.13 Limited exposure of weaver to market (LME)

2.2.14 Lack of awareness of targeted segment (LATS)

2.2.15 Socio-cultural challenges (SCC)

2.2.16 Involvement of exploitative middlemen in the supply chain (IEM)

\subsection{Success factors associated with handloom and carpet weaving}

Factors behind their success are numerous which has proliferated the growth of handloom and carpet weaving . Some of the common factors in various category are the market based criteria and the marketing strategies based criteria .

\subsubsection{Market based criteria}

2.3.1.1 Development and advancement of handloom-based technologies ( DAT);

2.3.1.2 Education and healthcare growth among weavers to sustain their livelihoods (EHCG);

2.3.1.3 Exposure to urban markets and targeted customer segments (EUM);

2.3.1.4 Partnerships with other organizations and the government (POG): for financial aid as well as technological developments,

2.3.1.5 Provision of ICT (PICT): and digital facilities such as wireless connectivity to the community;

2.3.1.6 Managing local communities (MLC): and building their capabilities for marketing,

2.3.1.7 Developing a structured ecosystem (DSE): for the organized operation of the production and other supply chain activities for proper regularization of the various sectors ;

2.3.1.8 Building the capacities of weavers (BCW): for organizing, producing efficiently, marketing and supply chain capabilities;

2.3.1.9 Enable the spirit of entrepreneurship (ESE)

2.3.2 Marketing strategies of community marketing efforts

2.3.2.1 Development of an e-commerce website for product sales $($ DoW);

2.3.2.2 Interaction with various organizations (IVO) : for exhibiting products to the urban customers segment;

2.3.2.3 Business marketing (BM) : for marketing products to organizational buyers;

2.3.2.4 Social marketing (SM) : for social upliftment of the local community;

2.3.2.5 Designing and implementing the marketing mix (DIMM)

2.3.2.6 Developing local communities and their skills $(D L C)$; and

2.3.2.7 Managing an ecosystem of partnerships (EoP) : with other NGOs, the government, the local community and other stakeholders. 


\section{INTERPRETIVE STRUCTURAL MODELLING METHODOLOGY}

Interpretive structural modelling methodology or ISM [15] is a known technique to map the relationships amongst the relevant elements as per decision maker's problems in a hierarchical manner. Starting with the identification of elements, it proceeds with establishing the contextual relationships between elements (by examining them in pairs ) and move on towards developing the structural selfinteraction (SSIM) matrix using VAXO [15] and then initial reachability matrix and final reachability matrix and rearranging the elements in topological order using the level partition matrices. A Mic-Mac analysis is performed afterwards which categorize the variables as per the driving and dependence power in to autonomous, dependent, driver and linkage category. Finally, a diagraph can be obtained.

\section{DEVELOPMENT OF ISM MODEL : CASE EXAMPLE}

\subsection{Barriers or challenges for handloom and carpet weaving sector}

The 16 barriers viz. Monotonous designs (MD); Expensive methods of designing (EMD) ; In appropriate calculation of raw material requirements (ICRM); Presence of manual errors (PME); Limited varieties (LV) ; Repetitions of designs (RoD); Storage and handling problems (SHP); Violation of Intellectual property rights (VIPR); Time factor/ Delay (TF) ; Infrastructural constraints (IC) ; Low use of technologies (LUT); Low wages to workers (LWW) ; Limited exposure of weaver to market (LME); Lack of awareness of targeted segment (LATS); Socio-cultural challenges (SCC) ; Involvement of exploitative middlemen in the supply chain (IEM) are now studied for possible inter-relationship amongst them using ISM methodology

\subsubsection{Construction of Structural self-interaction Matrix (SSIM)}

This matrix gives the pair-wise relationship between two variables i.e. I and $j$ based on VAXO. SSIM has been presented below in Fig 1.

\subsubsection{Construction of Initial Reachability Matrix and final reachability matrix}

The SSIM has been converted in to a binary matrix called the initial reachability matrix shown in fig. 2 by substituting $\mathrm{V}, \mathrm{A}$, $\mathrm{X}, \mathrm{O}$ by 1 or 0 as per the case. After incorporating the transitivity, the final reachability matrix is shown below in the Fig 3 .

\subsection{Success factors for handloom and carpet weaving sector [11-14]}

The 16 metrics viz. Development and advancement of handloom-based technologies (DAT); Education and healthcare growth among weavers to sustain their livelihoods (EHCG); Exposure to urban markets and targeted customer segments (EUM); Partnerships with other organizations and the government (POG); Provision of ICT (PICT); Managing local communities (MLC); Developing a structured ecosystem (DSE) ; Building the capacities of weavers (BCW) ; Enable the spirit of entrepreneurship (ESE); Development of an $e$ commerce website for product sales (DoW); Interaction with various organizations (IVO); Business marketing (BM); Social marketing (SM); Designing and implementing the marketing mix (DIMM) ; Managing NGO partnerships in skills development work (MP) ; Developing local communities and their skills (DLC) and Managing an ecosystem of partnerships (EoP) are now studied for possible inter-relationship amongst them using ISM methodology .

\subsubsection{Construction of Structural self-interaction Matrix (SSIM)}

This matrix gives the pair-wise relationship between two variables i.e. I and $j$ based on VAXO. SSIM has been presented below in Fig 4.

\subsubsection{Construction of Initial Reachability Matrix and final reachability matrix}

The SSIM has been converted in to a binary matrix called the initial reachability matrix shown in fig. 5 by substituting $\mathrm{V}, \mathrm{A}$, $\mathrm{X}, \mathrm{O}$ by 1 or 0 as per the case. After incorporating the transitivity, the final reachability matrix is shown below in the Fig.6

\begin{tabular}{|c|c|c|c|c|c|c|c|c|c|c|c|c|c|c|c|c|c|}
\hline S. No. & Barriers & 1 & 2 & 3 & 4 & 5 & 6 & 7 & 8 & 9 & 10 & 11 & 12 & 13 & 14 & 15 & 16 \\
\hline & & $\mathrm{MD}$ & $\begin{array}{c}\text { EM } \\
\text { D }\end{array}$ & $\begin{array}{l}\mathrm{IC} \\
\mathrm{R} \\
\mathrm{M}\end{array}$ & $\begin{array}{c}\mathrm{P} \\
\mathrm{M} \\
\mathrm{E}\end{array}$ & $\mathrm{LV}$ & RoD & SHP & $\begin{array}{c}\text { VIP } \\
\text { R }\end{array}$ & TF & IC & $\begin{array}{c}\mathrm{LU} \\
\mathrm{T}\end{array}$ & $\begin{array}{c}\text { LW } \\
\text { W }\end{array}$ & $\begin{array}{c}\mathrm{LM} \\
\mathrm{E}\end{array}$ & $\begin{array}{l}\text { LA } \\
\text { TS }\end{array}$ & $\begin{array}{c}\text { SC } \\
\text { C }\end{array}$ & $\begin{array}{l}\text { IE } \\
\text { M }\end{array}$ \\
\hline 1 & MD & & A & A & $\mathrm{V}$ & $\mathrm{X}$ & $\mathrm{V}$ & A & $\mathrm{O}$ & $\mathrm{O}$ & A & A & A & A & A & $\mathrm{A}$ & A \\
\hline 2 & EMD & & & $\mathrm{O}$ & $\mathrm{O}$ & $\mathrm{V}$ & $\mathrm{V}$ & $\mathrm{V}$ & $\mathrm{V}$ & $\mathrm{V}$ & $\mathrm{A}$ & $\mathrm{V}$ & $\mathrm{V}$ & $\mathrm{V}$ & $\mathrm{V}$ & $\mathrm{V}$ & $\mathrm{V}$ \\
\hline 3 & ICRM & & & & $\mathrm{A}$ & $\mathrm{V}$ & $\mathrm{V}$ & $\mathrm{X}$ & $\mathrm{O}$ & $\mathrm{V}$ & $\mathrm{A}$ & A & $\mathrm{A}$ & A & $\mathrm{A}$ & $\mathrm{A}$ & A \\
\hline 4 & PME & & & & & $\mathrm{A}$ & A & A & A & $\mathrm{A}$ & $\mathrm{A}$ & $\mathrm{A}$ & $\mathrm{A}$ & A & $\mathrm{A}$ & $\mathrm{A}$ & $\mathrm{A}$ \\
\hline 5 & LV & & & & & & $\mathrm{V}$ & A & A & $\mathrm{A}$ & $\mathrm{A}$ & A & A & A & $\mathrm{A}$ & $\mathrm{A}$ & A \\
\hline 6 & RoD & & & & & & & $\mathrm{O}$ & $\mathrm{O}$ & $\mathrm{A}$ & $\mathrm{A}$ & A & A & A & $\mathrm{A}$ & $\mathrm{A}$ & A \\
\hline 7 & SHP & & & & & & & & $\mathrm{O}$ & $\mathrm{V}$ & A & V & A & A & A & A & A \\
\hline 8 & VIPR & & & & & & & & & $\mathrm{O}$ & $\mathrm{A}$ & $\mathrm{A}$ & $\mathrm{V}$ & $\mathrm{A}$ & $\mathrm{A}$ & $\mathrm{A}$ & $\mathrm{A}$ \\
\hline 9 & $\mathrm{TF}$ & & & & & & & & & & $\mathrm{A}$ & A & A & $\mathrm{A}$ & A & $\mathrm{A}$ & A \\
\hline 10 & IC & & & & & & & & & & & V & $\mathrm{V}$ & $\mathrm{V}$ & $\mathrm{V}$ & $\mathrm{V}$ & V \\
\hline 11 & LUT & & & & & & & & & & & & $\mathrm{V}$ & $\mathrm{X}$ & $\mathrm{X}$ & $\mathrm{A}$ & A \\
\hline 12 & LWW & & & & & & & & & & & & & A & A & A & A \\
\hline
\end{tabular}




\begin{tabular}{|c|c|l|l|l|l|l|l|l|l|l|l|l|l|l|c|c|c|}
\hline 13 & LME & & & & & & & & & & & & & & A & A & A \\
\hline 14 & LATS & & & & & & & & & & & & & & & A & A \\
\hline 15 & SCC & & & & & & & & & & & & & & & & A \\
\hline 16 & IEM & & & & & & & & & & & & & & & & \\
\hline
\end{tabular}

Fig 1: SSIM matrix for pair wise relationship amongst barriers

\begin{tabular}{|c|c|c|c|c|c|c|c|c|c|c|c|c|c|c|c|c|c|}
\hline S. No. & Barriers & 1 & 2 & 3 & 4 & 5 & 6 & 7 & 8 & 9 & 10 & 11 & 12 & 13 & 14 & 15 & 16 \\
\hline & & $\mathrm{MD}$ & $\begin{array}{c}\text { EM } \\
\text { D }\end{array}$ & $\begin{array}{c}\mathrm{IC} \\
\mathrm{R} \\
\mathrm{M}\end{array}$ & $\begin{array}{c}\text { PM } \\
\text { E }\end{array}$ & $\mathrm{LV}$ & RoD & SHP & $\begin{array}{c}\text { VIP } \\
\text { R }\end{array}$ & TF & IC & $\begin{array}{c}\mathrm{LU} \\
\mathrm{T}\end{array}$ & $\begin{array}{c}\mathrm{LW} \\
\mathrm{W}\end{array}$ & $\begin{array}{c}\text { LM } \\
\text { E }\end{array}$ & $\begin{array}{l}\text { LA } \\
\text { TS }\end{array}$ & $\begin{array}{c}\mathrm{SC} \\
\mathrm{C}\end{array}$ & $\begin{array}{l}\mathrm{IE} \\
\mathrm{M}\end{array}$ \\
\hline 1 & MD & 1 & 0 & 0 & 1 & 1 & 1 & 0 & 0 & 0 & 0 & 0 & 0 & 0 & 0 & 0 & 0 \\
\hline 2 & EMD & 1 & 1 & 0 & 0 & 1 & 1 & 1 & 1 & 1 & 0 & 1 & 1 & 1 & 1 & 1 & 1 \\
\hline 3 & ICRM & 1 & 0 & 1 & 0 & 1 & 1 & 1 & 0 & 1 & 0 & 0 & 0 & 0 & 0 & 0 & 0 \\
\hline 4 & PME & 0 & 0 & 1 & 1 & 0 & 0 & 0 & 0 & 0 & 0 & 0 & 0 & 0 & 0 & 0 & 0 \\
\hline 5 & LV & 1 & 0 & 0 & 1 & 1 & 1 & 0 & 0 & 0 & 0 & 0 & 0 & 0 & 0 & 0 & 0 \\
\hline 6 & RoD & 0 & 0 & 0 & 1 & 0 & 1 & 0 & 0 & 0 & 0 & 0 & 0 & 0 & 0 & 0 & 0 \\
\hline 7 & SHP & 1 & 0 & 1 & 1 & 1 & 0 & 1 & 0 & 1 & 0 & 1 & 0 & 0 & 0 & 0 & 0 \\
\hline 8 & VIPR & 0 & 0 & 0 & 1 & 1 & 0 & 0 & 1 & 0 & 0 & 1 & 0 & 0 & 0 & 0 & 0 \\
\hline 9 & $\mathrm{TF}$ & 1 & 0 & 0 & 1 & 1 & 1 & 0 & 0 & 1 & 0 & 0 & 0 & 0 & 0 & 0 & 0 \\
\hline 10 & IC & 1 & 1 & 1 & 1 & 1 & 1 & 1 & 1 & 1 & 1 & 1 & 1 & 1 & 1 & 1 & 1 \\
\hline 11 & LUT & 1 & 0 & 1 & 1 & 1 & 1 & 0 & 1 & 1 & 0 & 1 & 1 & 1 & 1 & 0 & 0 \\
\hline 12 & LWW & 1 & 0 & 1 & 1 & 1 & 1 & 1 & 0 & 1 & 0 & 0 & 1 & 0 & 0 & 0 & 0 \\
\hline 13 & LME & 1 & 0 & 1 & 1 & 1 & 1 & 1 & 1 & 1 & 0 & 1 & 1 & 1 & 0 & 0 & 0 \\
\hline 14 & LATS & 1 & 0 & 1 & 1 & 1 & 1 & 1 & 1 & 1 & 0 & 1 & 1 & 1 & 1 & 0 & 0 \\
\hline 15 & SCC & 1 & 0 & 1 & 1 & 1 & 1 & 1 & 1 & 1 & 0 & 1 & 1 & 1 & 1 & 1 & 0 \\
\hline 16 & IEM & 1 & 0 & 1 & 1 & 1 & 1 & 1 & 1 & 1 & 0 & 1 & 1 & 1 & 1 & 1 & 1 \\
\hline
\end{tabular}

Fig 2: Initial reachability matrix

\begin{tabular}{|c|c|c|c|c|c|c|c|c|c|c|c|c|c|c|c|c|c|c|}
\hline S. No. & Barriers & 1 & 2 & 3 & 4 & 5 & 6 & 7 & 8 & 9 & 10 & 11 & 12 & 13 & 14 & 15 & 16 & \\
\hline & & $\mathrm{MD}$ & $\begin{array}{c}\text { EM } \\
\text { D }\end{array}$ & $\begin{array}{c}\text { IC } \\
\mathrm{R} \\
\mathrm{M}\end{array}$ & $\begin{array}{c}\mathrm{PM} \\
\mathrm{E}\end{array}$ & $\mathrm{LV}$ & $\begin{array}{c}\text { Ro } \\
\text { D }\end{array}$ & SHP & $\begin{array}{c}\text { VIP } \\
\text { R }\end{array}$ & $\mathrm{TF}$ & IC & $\begin{array}{c}\mathrm{LU} \\
\mathrm{T}\end{array}$ & $\begin{array}{c}\text { LW } \\
\text { W }\end{array}$ & $\begin{array}{c}\mathrm{LM} \\
\mathrm{E}\end{array}$ & $\begin{array}{c}\mathrm{L} \\
\mathrm{A} \\
\mathrm{TS}\end{array}$ & $\begin{array}{c}\mathrm{SC} \\
\mathrm{C}\end{array}$ & $\begin{array}{l}\mathrm{IE} \\
\mathrm{M}\end{array}$ & $\begin{array}{l}\text { D. } \\
\text { P }\end{array}$ \\
\hline 1 & MD & 1 & 0 & 1 & 1 & 1 & 1 & 0 & 0 & 0 & 0 & 0 & 0 & 0 & 0 & 0 & 0 & 6 \\
\hline 2 & EMD & 1 & 1 & 1 & 1 & 1 & 1 & 1 & 1 & 1 & 0 & 1 & 1 & 1 & 1 & 1 & 1 & 14 \\
\hline 3 & ICRM & 1 & 0 & 1 & 1 & 1 & 1 & 1 & 0 & 1 & 0 & 0 & 0 & 0 & 0 & 0 & 0 & 7 \\
\hline 4 & PME & 1 & 0 & 1 & 1 & 1 & 1 & 1 & 0 & 1 & 0 & 0 & 0 & 0 & 0 & 0 & 0 & 7 \\
\hline 5 & $\mathrm{LV}$ & 1 & 0 & 1 & 1 & 1 & 1 & 0 & 0 & 1 & 0 & 0 & 0 & 0 & 0 & 0 & 0 & 6 \\
\hline 6 & RoD & 0 & 0 & 1 & 1 & 0 & 1 & 0 & 0 & 0 & 0 & 0 & 0 & 0 & 0 & 0 & 0 & 3 \\
\hline 7 & SHP & 1 & 0 & 1 & 1 & 1 & 1 & 1 & 0 & 1 & 0 & 1 & 0 & 0 & 0 & 0 & 0 & 7 \\
\hline 8 & VIPR & 1 & 0 & 1 & 1 & 1 & 1 & 1 & 1 & 1 & 0 & 1 & 1 & 0 & 0 & 0 & 0 & 8 \\
\hline 9 & $\mathrm{TF}$ & 1 & 0 & 1 & 1 & 1 & 1 & 0 & 0 & 1 & 0 & 0 & 0 & 0 & 0 & 0 & 0 & 6 \\
\hline 10 & IC & 1 & 1 & 1 & 1 & 1 & 1 & 1 & 1 & 1 & 1 & 1 & 1 & 1 & 1 & 1 & 1 & 16 \\
\hline 11 & LUT & 1 & 0 & 1 & 1 & 1 & 1 & 1 & 1 & 1 & 0 & 1 & 1 & 1 & 1 & 0 & 0 & 13 \\
\hline
\end{tabular}




\begin{tabular}{|c|c|c|c|c|c|c|c|c|c|c|c|c|c|c|c|c|c|c|}
\hline 12 & LWW & 1 & 0 & 1 & 1 & 1 & 1 & 1 & 0 & 1 & 0 & 0 & 1 & 0 & 0 & 0 & 0 & 8 \\
\hline 13 & LME & 1 & 0 & 1 & 1 & 1 & 1 & 1 & 1 & 1 & 0 & 1 & 1 & 1 & 1 & 0 & 0 & 12 \\
\hline 14 & LATS & 1 & 0 & 1 & 1 & 1 & 1 & 1 & 1 & 1 & 0 & 1 & 1 & 1 & 1 & 0 & 0 & 12 \\
\hline 15 & SCC & 1 & 0 & 1 & 1 & 1 & 1 & 1 & 1 & 1 & 0 & 1 & 1 & 1 & 1 & 1 & 0 & 13 \\
\hline 16 & IEM & 1 & 0 & 1 & 1 & 1 & 1 & 1 & 1 & 1 & 0 & 1 & 1 & 1 & 1 & 1 & 1 & 14 \\
\hline & De.P & 15 & 2 & 16 & 16 & 15 & 16 & 12 & 8 & 14 & 1 & 9 & 10 & 7 & 7 & 4 & 3 & \\
\hline
\end{tabular}

Fig 3 : Final reachability matrix

D.P : Driving power ;De.P : dependence power

\begin{tabular}{|c|c|c|c|c|c|c|c|c|c|c|c|c|c|c|c|c|c|}
\hline S. No. & Barriers & 1 & 2 & 3 & 4 & 5 & 6 & 7 & 8 & 9 & 10 & 11 & 12 & 13 & 14 & 15 & 16 \\
\hline & & DAT & $\begin{array}{c}\text { EHC } \\
\text { G }\end{array}$ & $\begin{array}{c}\text { EU } \\
M\end{array}$ & POG & $\begin{array}{c}\text { PIC } \\
\text { T }\end{array}$ & MLC & DSE & $\begin{array}{c}\text { BC } \\
W\end{array}$ & $\begin{array}{c}\text { ES } \\
\text { E }\end{array}$ & $\begin{array}{l}\text { Do } \\
\text { W }\end{array}$ & $\begin{array}{c}\text { IV } \\
\text { O }\end{array}$ & $\mathrm{BM}$ & SM & $\begin{array}{l}\text { DI } \\
\mathrm{M} \\
\mathrm{M}\end{array}$ & $\begin{array}{c}\text { DL } \\
\text { C }\end{array}$ & $\begin{array}{c}\text { Eo } \\
P\end{array}$ \\
\hline 1 & DAT & & $\mathrm{A}$ & $\mathrm{A}$ & $\bar{A}$ & $\mathrm{~A}$ & A & A & $\mathrm{A}$ & $\mathrm{A}$ & $\mathrm{A}$ & $\mathrm{A}$ & A & $\bar{A}$ & $\mathrm{~A}$ & $\mathrm{~A}$ & $\mathrm{~A}$ \\
\hline 2 & EHCG & & & $\mathrm{A}$ & $\mathrm{A}$ & $\mathrm{A}$ & $\mathrm{A}$ & $\mathrm{A}$ & $\mathrm{A}$ & $\mathrm{A}$ & $\mathrm{A}$ & $\mathrm{A}$ & $\mathrm{A}$ & $\mathrm{A}$ & $\mathrm{A}$ & $\mathrm{A}$ & $\mathrm{A}$ \\
\hline 3 & EUM & & & & $\mathrm{V}$ & $\mathrm{V}$ & $\mathrm{V}$ & $\mathrm{V}$ & $\mathrm{V}$ & $\mathrm{V}$ & $\mathrm{V}$ & $\mathrm{V}$ & $\mathrm{V}$ & $\mathrm{V}$ & $\mathrm{V}$ & $\mathrm{V}$ & $\mathrm{V}$ \\
\hline 4 & POG & & & & & $\mathrm{A}$ & $\mathrm{O}$ & $\mathrm{V}$ & $\mathrm{V}$ & $\mathrm{V}$ & $\mathrm{V}$ & $\mathrm{V}$ & $\mathrm{V}$ & $\mathrm{V}$ & $\mathrm{V}$ & $\mathrm{V}$ & $\mathrm{V}$ \\
\hline 5 & PICT & & & & & & V & $\mathrm{V}$ & $\mathrm{V}$ & $\mathrm{V}$ & $\mathrm{V}$ & $\mathrm{V}$ & $\mathrm{V}$ & V & $\mathrm{V}$ & $\mathrm{V}$ & $\mathrm{V}$ \\
\hline 6 & MLC & & & & & & & V & $\mathrm{V}$ & $\mathrm{V}$ & $\mathrm{V}$ & $\mathrm{V}$ & $\mathrm{V}$ & V & $\mathrm{V}$ & $\mathrm{V}$ & $\mathrm{V}$ \\
\hline 7 & DSE & & & & & & & & $\mathrm{V}$ & $\mathrm{V}$ & $\mathrm{V}$ & $\mathrm{V}$ & $\mathrm{V}$ & $\mathrm{V}$ & $\mathrm{V}$ & $\mathrm{V}$ & $\mathrm{V}$ \\
\hline 8 & $\mathrm{BCW}$ & & & & & & & & & A & A & $\mathrm{V}$ & V & V & V & $\mathrm{V}$ & $\mathrm{V}$ \\
\hline 9 & ESE & & & & & & & & & & $\mathrm{V}$ & $\mathrm{V}$ & $\mathrm{V}$ & $\mathrm{V}$ & $\mathrm{V}$ & $\mathrm{V}$ & $\mathrm{V}$ \\
\hline 10 & DoW & & & & & & & & & & & A & A & A & A & A & A \\
\hline 11 & IVO & & & & & & & & & & & & A & $\mathrm{A}$ & $\mathrm{A}$ & $\mathrm{A}$ & $\mathrm{A}$ \\
\hline 12 & $\overline{B M}$ & & & & & & & & & & & & & $\mathrm{~A}$ & $\mathrm{~A}$ & $\mathrm{~A}$ & $\mathrm{~A}$ \\
\hline 13 & SM & & & & & & & & & & & & & & $\mathrm{V}$ & $\mathrm{V}$ & $\mathrm{V}$ \\
\hline V14 & DIMM & & & & & & & & & & & & & & & $\mathrm{V}$ & $\mathrm{V}$ \\
\hline 15 & DLC & & & & & & & & & & & & & & & & $\mathrm{V}$ \\
\hline 16 & EoP & & & & & & & & & & & & & & & & \\
\hline
\end{tabular}

Fig 4 : SSIM for success factors

\begin{tabular}{|c|c|c|c|c|c|c|c|c|c|c|c|c|c|c|c|c|c|}
\hline S. No. & Barriers & 1 & 2 & 3 & 4 & 5 & 6 & 7 & 8 & 9 & 10 & 11 & 12 & 13 & 14 & 15 & 16 \\
\hline & & DAT & $\begin{array}{c}\text { EHC } \\
\text { G }\end{array}$ & $\begin{array}{c}\text { EU } \\
\text { M }\end{array}$ & POG & $\begin{array}{c}\text { PIC } \\
\text { T }\end{array}$ & MLC & DSE & $\begin{array}{c}\text { BC } \\
\text { W }\end{array}$ & $\begin{array}{c}\text { ES } \\
\text { E }\end{array}$ & $\begin{array}{c}\text { Do } \\
\text { W }\end{array}$ & $\begin{array}{c}\text { IV } \\
\text { O }\end{array}$ & $\begin{array}{c}\text { BM } \\
\text { SM }\end{array}$ & $\begin{array}{c}\text { DI } \\
\text { M } \\
\text { M }\end{array}$ & $\begin{array}{c}\text { DL } \\
\text { C } \\
\text { P }\end{array}$ \\
\hline 1 & DAT & 1 & 0 & 0 & 0 & 0 & 0 & 0 & 0 & 0 & 0 & 0 & 0 & 0 & 0 & 0 & 0 \\
\hline 2 & EHCG & 1 & 1 & 0 & 0 & 0 & 0 & 0 & 0 & 0 & 0 & 0 & 0 & 0 & 0 & 0 & 0 \\
\hline 3 & EUM & 1 & 1 & 1 & 1 & 1 & 1 & 1 & 1 & 1 & 1 & 1 & 1 & 1 & 1 & 1 & 1 \\
\hline 4 & POG & 1 & 1 & 0 & 1 & 0 & 0 & 1 & 1 & 1 & 1 & 1 & 1 & 1 & 1 & 1 & 1 \\
\hline 5 & PICT & 1 & 1 & 0 & 1 & 1 & 1 & 1 & 1 & 1 & 1 & 1 & 1 & 1 & 1 & 1 & 1 \\
\hline 6 & MLC & 1 & 1 & 0 & 0 & 0 & 1 & 1 & 1 & 1 & 1 & 1 & 1 & 1 & 1 & 1 & 1 \\
\hline 7 & DSE & 1 & 1 & 0 & 1 & 0 & 0 & 1 & 1 & 1 & 1 & 1 & 1 & 1 & 1 & 1 & 1 \\
\hline 8 & BCW & 1 & 1 & 0 & 1 & 0 & 0 & 0 & 1 & 0 & 0 & 1 & 1 & 1 & 1 & 1 & 1 \\
\hline
\end{tabular}




\begin{tabular}{|c|c|c|c|c|c|c|c|c|c|c|c|c|c|c|c|c|c|}
\hline 9 & ESE & 1 & 1 & 0 & 1 & 0 & 0 & 0 & 1 & 1 & 1 & 1 & 1 & 1 & 1 & 1 & 1 \\
\hline 10 & DoW & 1 & 1 & 0 & 1 & 0 & 0 & 0 & 1 & 0 & 1 & 0 & 0 & 0 & 0 & 0 & 0 \\
\hline 11 & IVO & 1 & 1 & 0 & 1 & 0 & 0 & 0 & 0 & 0 & 1 & 1 & 0 & 0 & 0 & 0 & 0 \\
\hline 12 & BM & 1 & 1 & 0 & 1 & 0 & 0 & 0 & 0 & 0 & 1 & 1 & 1 & 0 & 0 & 0 & 0 \\
\hline 13 & SM & 1 & 1 & 0 & 1 & 0 & 0 & 0 & 0 & 0 & 1 & 1 & 1 & 1 & 1 & 1 & 1 \\
\hline 14 & DIMM & 1 & 1 & 0 & 1 & 0 & 0 & 0 & 0 & 0 & 1 & 1 & 1 & 0 & 1 & 1 & 1 \\
\hline 15 & DLC & 1 & 1 & 0 & 1 & 0 & 0 & 0 & 0 & 0 & 1 & 1 & 1 & 0 & 0 & 1 & 1 \\
\hline 16 & EoP & 1 & 1 & 0 & 1 & 0 & 0 & 0 & 0 & 0 & 1 & 1 & 1 & 0 & 0 & 0 & 1 \\
\hline
\end{tabular}

Fig 5: Initial reachability matrix for success factors

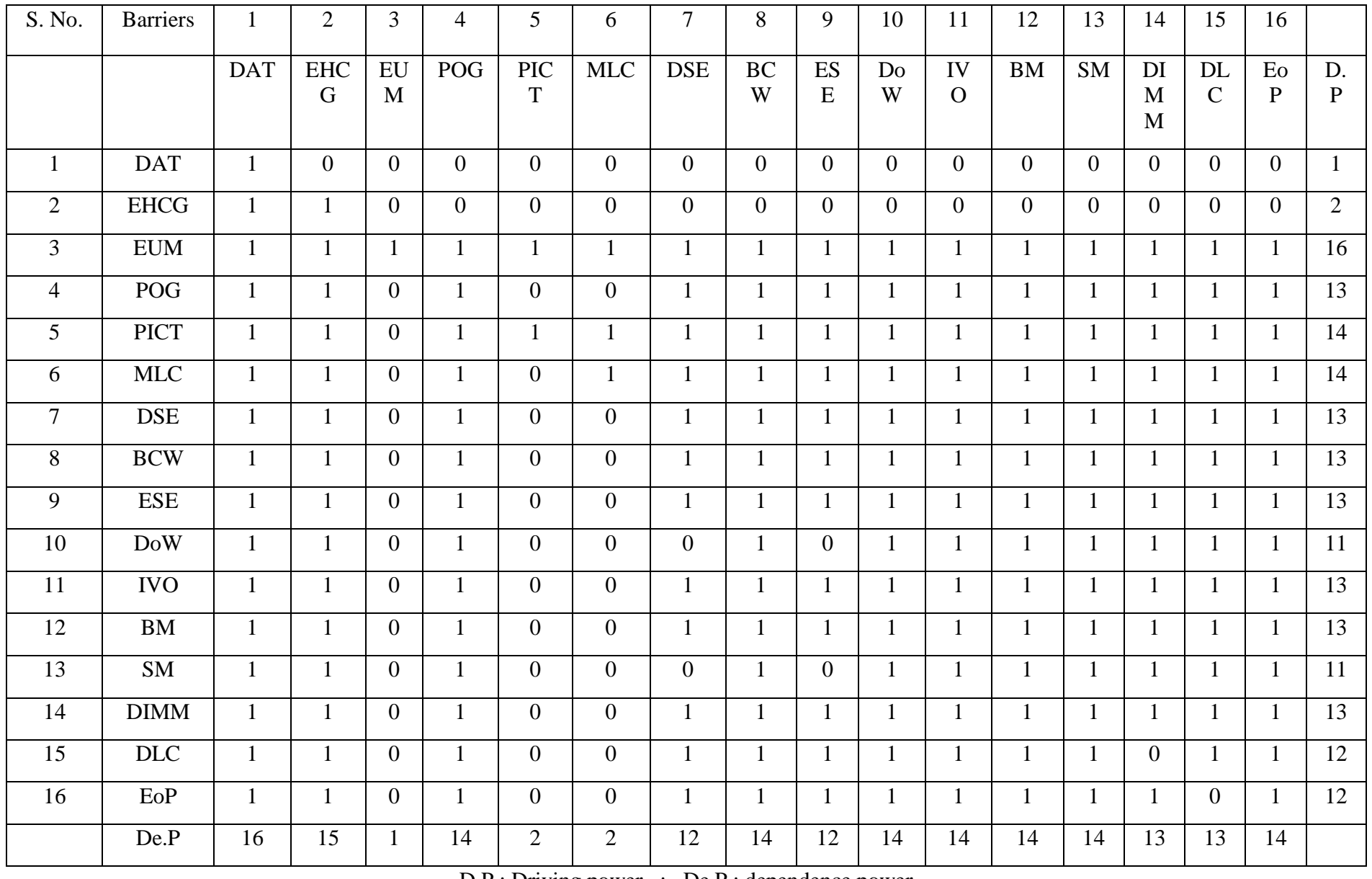

\section{D.P : Driving power ; De.P : dependence power}

Fig 6 : final reachability matrix for success factors

\subsubsection{Level Partition [ for barriers]}

From the final reachability matrix, reachability and final antecedent set for each factor are found. The elements for which the reachability and intersection sets are same are the top-level element in the ISM hierarchy. After the identification of top level element, it is separated out from the other elements and the process continues for next level of elements. Reachability set, antecedent set, intersection set along with different level for elements have been shown below in table 1 .

Table 4.1.3.1: Iteration I

\begin{tabular}{|c|c|c|c|c|}
\hline $\begin{array}{c}\text { S. } \\
\text { No }\end{array}$ & $\begin{array}{c}\text { Reachability } \\
\text { set }\end{array}$ & Antecedent set & $\begin{array}{c}\text { Intersectio } \\
\text { n set }\end{array}$ & $\begin{array}{c}\text { Lev } \\
\text { el }\end{array}$ \\
\hline
\end{tabular}

\begin{tabular}{|c|c|c|c|c|}
\hline 1 & $3,4,6$ & $\begin{array}{c}1,2,3,4,5,6,7,8,9 \\
10,11,12,13,14 \\
15,16\end{array}$ & $3,4,6$ & \\
\hline 2 & $1,3,4,5,6$ & $\begin{array}{c}1,2,3,4,5,7,8,9 \\
10,11,12,13,14,1 \\
5,16\end{array}$ & $1,3,4,5$ & \\
\hline 3 & $1,3,4,5,6,9$ & $\begin{array}{l}2,3,4,5,7,8,9,10,1 \\
1,12,13,14,15,16\end{array}$ & $3,4,5,9$ & I \\
\hline 4 & $1,3,4,5,6,7,9$ & $\begin{array}{l}2,3,4,7,8,9,10, \\
11,12,13,14,15\end{array}$ & $3,4,7,9$ & \\
\hline
\end{tabular}




\begin{tabular}{|c|c|c|c|}
\hline & & 16 & \\
\hline 5 & $1,3,4,5,6,7,9$, & $2,8,10,11,12,13,1$ & 12 \\
& 12 & $4,15,16$ & \\
\hline 6 & $1,3,4,5,6,7,9$, & $2,8,10,11,13,14,1$ & 11 \\
& 11,12 & 5,16 & \\
\hline 7 & $1,3,4,5,6,7,8$, & $2,8,10,11,13,14,1$ & 8,11 \\
& $9,11,12$ & 5,16 & \\
\hline 8 & $1,3,4,5,6,7,8$, & $2,8,10,11,13,14,1$ & $8,11,13,14$ \\
& $9,11,12,13,1$ & 5,16 & \\
& 4 & & \\
\hline 9 & $1,2,3,4,5,6,7$, & $2,8,10,11,13,14,1$ & $8,11,13,14$, \\
& $8,9,11,12$, & 5,16 & 15 \\
& $13,14,15$ & & \\
& $1,2,3,4,5,6,7$, & $2,8,10,11,13,14,1$ & $2,8,11,13,1$ \\
& $8,9,11,12$, & 5,16 & $4,15,16$ \\
& $13,14,15,16$ & & \\
& & & $4,15,16$ \\
\hline 11 & $1,2,3,4,5,6,7$, & $2,8,10,11,13,14,1$ & $2,8,11,13,1$ \\
& $8,9,11,12,13$, & 5,16 & \\
& $14,15,16$ & & \\
\hline
\end{tabular}

Table 4.1.3.2: Iteration II

\begin{tabular}{|c|c|c|c|c|}
\hline $\begin{array}{l}\text { S. } \\
\text { No }\end{array}$ & $\begin{array}{c}\text { Reachability } \\
\text { set }\end{array}$ & Antecedent set & $\begin{array}{l}\text { Intersectio } \\
\text { n set }\end{array}$ & $\begin{array}{c}\text { Lev } \\
\text { el }\end{array}$ \\
\hline 2 & 1,5 & $\begin{array}{c}1,2,5,7,8,9,10,11 \\
12,13,14 \\
15,16\end{array}$ & 1,5 & \multirow{10}{*}{ II } \\
\hline 3 & $1,5,9$ & $\begin{array}{c}2,5,7,8,9,10,11,1 \\
2,13,14,15,16\end{array}$ & 5,9 & \\
\hline 4 & $1,5,7,9$ & $\begin{array}{c}2,7,8,9,10,11,12 \\
13,14,15,16\end{array}$ & 7,9 & \\
\hline 5 & $1,5,7,9,12$ & $\begin{array}{c}2,8,10,11,12,13,1 \\
4,15,16\end{array}$ & 12 & \\
\hline 6 & $1,5,7,9,11,12$ & $\begin{array}{c}2,8,10,11,13,14,1 \\
5,16\end{array}$ & 11 & \\
\hline 7 & $\begin{array}{c}1,5,7,8,9,11 \\
12\end{array}$ & $\begin{array}{c}2,8,10,11,13,14,1 \\
5,16\end{array}$ & 8,11 & \\
\hline 8 & $\begin{array}{c}1,5,7,8,9,11 \\
12,13,14\end{array}$ & $\begin{array}{c}2,8,10,11,13,14,1 \\
5,16\end{array}$ & $8,11,13,14$ & \\
\hline 9 & $\begin{array}{c}1,2,5,7,8,9,1 \\
1,12,13,14 \\
15\end{array}$ & $\begin{array}{c}2,8,10,11,13,14,1 \\
5,16\end{array}$ & $\begin{array}{c}8,11,13,14 \\
15\end{array}$ & \\
\hline 10 & $\begin{array}{c}1,2,5,7,8,9,1 \\
1,12,13,14 \\
15,16\end{array}$ & $\begin{array}{c}2,8,10,11,13,14,1 \\
5,16\end{array}$ & $\begin{array}{c}2,8,11,13,1 \\
4,15,16\end{array}$ & \\
\hline 11 & $\begin{array}{c}1,2,5,7,8,9,1 \\
1,12,13,14 \\
15,16\end{array}$ & $\begin{array}{c}2,8,10,11,13,14,1 \\
5,16\end{array}$ & $\begin{array}{c}2,8,11,13,1 \\
4,15,16\end{array}$ & \\
\hline
\end{tabular}

Table 4.1.3.3: Iteration III

\begin{tabular}{|c|c|c|c|c|}
\hline $\begin{array}{l}\text { S. } \\
\text { No }\end{array}$ & $\begin{array}{c}\text { Reachability } \\
\text { set }\end{array}$ & Antecedent set & $\begin{array}{l}\text { Intersectio } \\
\text { n set }\end{array}$ & $\begin{array}{c}\text { Lev } \\
\text { el }\end{array}$ \\
\hline 3 & 9 & $\begin{array}{c}2,7,8,9,10,11,12 \\
13,14,15,16\end{array}$ & 9 & \multirow{9}{*}{ III } \\
\hline 4 & 7,9 & $\begin{array}{c}2,7,8,9,10,11,12 \\
13,14,15,16\end{array}$ & 7,9 & \\
\hline 5 & $7,9,12$ & $\begin{array}{c}2,8,10,11,12,13,1 \\
4,15,16\end{array}$ & 12 & \\
\hline 6 & $7,9,11,12$ & $\begin{array}{c}2,8,10,11,13,14,1 \\
5,16\end{array}$ & 11 & \\
\hline 7 & $7,8,9,11,12$ & $\begin{array}{c}2,8,10,11,13,14,1 \\
5,16\end{array}$ & 8,11 & \\
\hline 8 & $\begin{array}{c}7,8,9,11,12,1 \\
3,14\end{array}$ & $\begin{array}{c}2,8,10,11,13,14,1 \\
5,16\end{array}$ & $8,11,13,14$ & \\
\hline 9 & $\begin{array}{c}2,7,8,9,11,12 \\
, 13,14,15\end{array}$ & $\begin{array}{c}2,8,10,11,13,14,1 \\
5,16\end{array}$ & $\begin{array}{c}8,11,13,14 \\
15\end{array}$ & \\
\hline 10 & $\begin{array}{r}2,7,8,9,11,12 \\
, 13,14,15,16\end{array}$ & $\begin{array}{c}2,8,10,11,13,14,1 \\
5,16\end{array}$ & $\begin{array}{c}2,8,11,13,1 \\
4,15,16\end{array}$ & \\
\hline 11 & $\begin{array}{r}2,7,8,9,11,12 \\
, 13,14,15,16\end{array}$ & $\begin{array}{c}2,8,10,11,13,14,1 \\
5,16\end{array}$ & $\begin{array}{c}2,8,11,13,1 \\
4,15,16\end{array}$ & \\
\hline
\end{tabular}

Table 4.1.3.4: Iteration IV

\begin{tabular}{|c|c|c|c|c|}
\hline $\begin{array}{l}\text { S. } \\
\text { No }\end{array}$ & $\begin{array}{c}\text { Reachability } \\
\text { set }\end{array}$ & Antecedent set & $\begin{array}{c}\text { Intersectio } \\
\text { n set }\end{array}$ & $\begin{array}{c}\text { Lev } \\
\text { el }\end{array}$ \\
\hline 5 & 12 & $\begin{array}{c}2,8,10,11,12,13,1 \\
4,15,16\end{array}$ & 12 & \multirow{7}{*}{ IV } \\
\hline 6 & 11,12 & $\begin{array}{c}2,8,10,11,13,14,1 \\
5,16\end{array}$ & 11 & \\
\hline 7 & $8,11,12$ & $\begin{array}{c}2,8,10,11,13,14,1 \\
5,16\end{array}$ & 8,11 & \\
\hline 8 & $\begin{array}{c}8,11,12,13,1 \\
4\end{array}$ & $\begin{array}{c}2,8,10,11,13,14,1 \\
5,16\end{array}$ & $8,11,13,14$ & \\
\hline 9 & $\begin{array}{c}2,8,11,12,13, \\
14,15\end{array}$ & $\begin{array}{c}2,8,10,11,13,14,1 \\
5,16\end{array}$ & $\begin{array}{c}8,11,13,14, \\
15\end{array}$ & \\
\hline 10 & $\begin{array}{c}2,8,11,12,13 \\
14,15,16\end{array}$ & $\begin{array}{c}2,8,10,11,13,14,1 \\
5,16\end{array}$ & $\begin{array}{c}2,8,11,13,1 \\
4,15,16\end{array}$ & \\
\hline 11 & $\begin{array}{c}2,8,11,12,13 \\
14,15,16\end{array}$ & $\begin{array}{c}2,8,10,11,13,14,1 \\
5,16\end{array}$ & $\begin{array}{c}2,8,11,13,1 \\
4,15,16\end{array}$ & \\
\hline
\end{tabular}

Table 4.1.3.5: Iteration V

\begin{tabular}{|c|c|c|c|c|}
\hline $\begin{array}{c}\text { S.No } \\
\cdot\end{array}$ & $\begin{array}{c}\text { Reachability } \\
\text { set }\end{array}$ & Antecedent set & $\begin{array}{c}\text { Intersectio } \\
\text { n set }\end{array}$ & $\begin{array}{c}\text { Lev } \\
\text { el }\end{array}$ \\
\hline 6 & 11 & $\begin{array}{c}2,8,10,11,13,14,1 \\
5,16\end{array}$ & 11 & \\
& & $\begin{array}{c}2,8,10,11,13,14,1 \\
5,16\end{array}$ & 8,11 & \\
\hline 7 & $\mathbf{8 , 1 1}$ & $2,8,10,11,13,14,1$ & $8,11,13,14$ & \\
\hline 8 & $8,11,12,13,1$ & \multicolumn{2}{|c}{} \\
\hline
\end{tabular}




\begin{tabular}{|c|c|c|c|c|}
\hline & 4 & 5,16 & & \\
\hline 9 & $2,8,11,12,13$, & $2,8,10,11,13,14,1$ & $8,11,13,14$, & $\mathbf{V}$ \\
& 14,15 & 5,16 & 15 & \\
\cline { 1 - 4 } 10 & $2,8,11,12,13$, & $2,8,10,11,13,14,1$ & $2,8,11,13,1$ & \\
& $14,15,16$ & 5,16 & $4,15,16$ & \\
\cline { 1 - 4 } 11 & $2,8,11,12,13$, & $2,8,10,11,13,14,1$ & $2,8,11,13,1$ & \\
& $14,15,16$ & 5,16 & $4,15,16$ & \\
& & & \\
\end{tabular}

\subsubsection{ISM Diagraph}

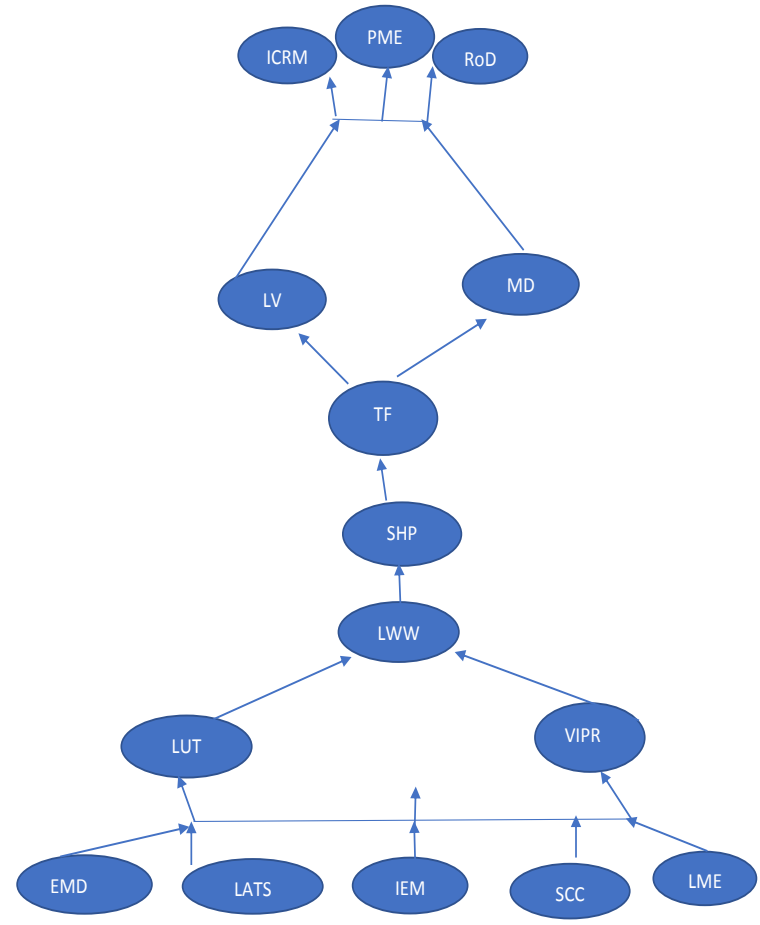

The critical success factors described earlier are classified in to four clusters viz. autonomous factor, dependent factors, linkage factors and independent / Driving factors are mentioned below.

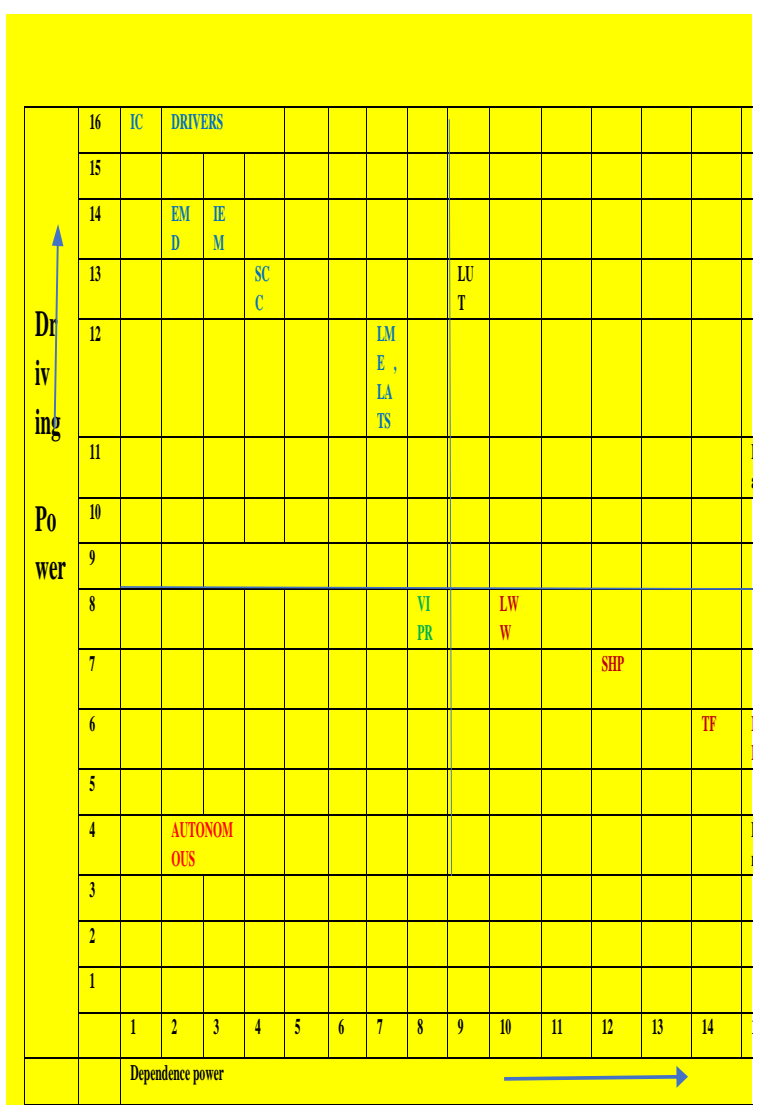

Fig. 4.Driving Power and Dependence Diagram

\begin{tabular}{|c|c|c|c|c|}
\hline $\begin{array}{c}\text { S.No } \\
\text {. }\end{array}$ & $\begin{array}{c}\text { Reachability } \\
\text { set }\end{array}$ & Antecedent set & $\begin{array}{c}\text { Intersectio } \\
\text { n set }\end{array}$ & $\begin{array}{c}\text { Lev } \\
\text { el }\end{array}$ \\
\hline 8 & 13,14 & $2,10,13,14,15,16$ & 13,14 & \\
\hline 9 & $2,13,14,15$ & $2,10,13,14,15,16$ & $13,14,15$ & \\
\hline 10 & $\begin{array}{c}2,13,14,15 \\
16\end{array}$ & $2,10,13,14,15,16$ & $\begin{array}{c}2,13,14,15, \\
16\end{array}$ & VI \\
\hline 11 & $\begin{array}{c}2,13,14,15 \\
16\end{array}$ & $2,10,13,14,15,16$ & $\begin{array}{c}2,13,14,15, \\
16\end{array}$ & \\
\hline
\end{tabular}

\subsubsection{Classification of factors}

\section{Fig 5. ISM Diagraph}

4.2.6 Level Partition [ for success factors]

For success factors as well the iterations are performed on the final reachability matrix shown in fig 6 as in section 4.1.3. Reachability set, antecedent set, intersection set along with different level for elements have been shown below in table4.2.3.1-4.2.3.7.

Table 4.2.3.1: Iteration I

\begin{tabular}{|c|c|c|c|c|}
\hline $\begin{array}{c}\text { S. } \\
\text { No }\end{array}$ & $\begin{array}{c}\text { Reachability } \\
\text { set }\end{array}$ & $\begin{array}{c}\text { Antecedent } \\
\text { set }\end{array}$ & $\begin{array}{c}\text { Intersectio } \\
\text { n set }\end{array}$ & $\begin{array}{c}\text { Lev } \\
\text { el }\end{array}$ \\
\hline $\mathbf{1}$ & $\mathbf{1}$ & $\begin{array}{c}\mathbf{1 , 2 , 3 , 4 , 5 , 6 , 7 , 8 ,} \\
\mathbf{9 , 1 0 , 1 1 , 1 2 , 1 3 ,} \\
\mathbf{1 4 , 1 5 , 1 6 ,}\end{array}$ & $\mathbf{1}$ & $\mathbf{1}$ \\
& & $2,3,4,5,6,7,8,9$, & 2 & 2 \\
\hline 2 & 1,2 & $10,11,12,13$, & & \\
& & $14,15,16$, & & \\
\hline 3 & $1,2,4,8,10$, & $3,4,5,6,7,8,9$, & $4,8,10,11,1$ & 3 \\
& $11,12,13,16$ & $10,11,12,13,14$ & $2,13,16$ & \\
& & $, 15,16$ & & 4 \\
\hline 4 & $1,2,4,8,10$, & $3,4,5,6,7,8,9$, & $4,8,10,11,1$ & 4 \\
& $11,12,13,15,16$ & $10,11,12,13,14$ & $2,13,15$ & \\
& &, 15 & & \\
\hline 5 & $1,2,4,8,10$, & $3,4,5,6,7,8,9$, & $4,8,10,11,1$ & 5 \\
& $11,12,13,14$, & $10,11,12,13,14$ & 2,13 & \\
& 15,16 & & & \\
\hline 6 & $1,2,4,7,9,8$, & $3,4,5,6,7,8,9,1$ & $4,7,9,8,11$, & 6 \\
& $10,11,12$, & $1,12,14$ & 12,14 & \\
& $13,14,15,16$ & & & \\
\hline 7 & $1,2,4,5,6,7,9,8$, & $3,5,6$ & 5,6 & 7 \\
& $10,11,12$ & & & \\
\hline & & & & \\
\hline
\end{tabular}




\begin{tabular}{|c|c|c|c|c|}
\hline & $, 13,14,15,16$ & & & \\
\hline 8 & $1,2,3,4,5,6,7,9$, & 3 & 3 & 8 \\
& $8,10,11,12,13,1$ & & & \\
& $4,15,16$ & & & \\
\hline
\end{tabular}

Table 4.2.3.2: Iteration II

\begin{tabular}{|c|c|c|c|c|}
\hline $\begin{array}{c}\text { S. } \\
\text { No } \\
\text {. }\end{array}$ & $\begin{array}{c}\text { Reachability } \\
\text { set }\end{array}$ & $\begin{array}{c}\text { Antecedent } \\
\text { set }\end{array}$ & $\begin{array}{c}\text { Intersectio } \\
\text { n set }\end{array}$ & $\begin{array}{c}\text { Lev } \\
\text { el }\end{array}$ \\
\hline 2 & 2 & $\begin{array}{c}2,3,4,5,6,7,8,9 \\
10,11,12 \\
13,14,15,16\end{array}$ & 2 & \\
\hline 3 & $\begin{array}{c}2,4,8,10,11,12 \\
13,16\end{array}$ & $\begin{array}{c}3,4,5,6,7,8,9,1 \\
0,11,12,13,14 \\
15,16\end{array}$ & $\begin{array}{c}4,8,10,11,1 \\
2,13,16\end{array}$ & \\
\hline 4 & $\begin{array}{c}2,4,8,10,11,12 \\
13,15,16\end{array}$ & $\begin{array}{c}3,4,5,6,7,8,9,1 \\
0,11,12,13,14 \\
15\end{array}$ & $\begin{array}{c}4,8,10,11,1 \\
2,13,15\end{array}$ & \\
\hline 5 & $\begin{array}{c}2,4,8,10,11,12 \\
13,14,15,16\end{array}$ & $\begin{array}{c}3,4,5,6,7,8,9,1 \\
0,11,12,13,14\end{array}$ & $\begin{array}{c}4,8,10,11,1 \\
2,13\end{array}$ & \\
\hline 6 & $\begin{array}{c}2,4,7,9,8,10,11 \\
12,13,14,15,16\end{array}$ & $\begin{array}{c}3,4,5,6,7,8,9,1 \\
1,12,14\end{array}$ & $\begin{array}{c}4,7,9,8,11 \\
12,14\end{array}$ & \\
\hline 7 & $\begin{array}{c}2,4,5,6,7,9,8,10 \\
, 11,12,13,14,15 \\
, 16\end{array}$ & $3,5,6$ & 5,6 & II \\
\hline 8 & $\begin{array}{c}2,3,4,5,6,7,9,8 \\
10,11,12,13,14 \\
15,16\end{array}$ & 3 & 3 & \\
\hline
\end{tabular}

Table 4.2.3.3: Iteration III

\begin{tabular}{|c|c|c|c|c|}
\hline $\begin{array}{l}\text { S. } \\
\text { No }\end{array}$ & Reachability set & $\begin{array}{c}\text { Anteceden } \\
\text { t set }\end{array}$ & $\begin{array}{c}\text { Intersectio } \\
\text { n set }\end{array}$ & $\begin{array}{c}\text { Lev } \\
\text { el }\end{array}$ \\
\hline 3 & $4,8,10,11,12,13,16$ & $\begin{array}{c}3,4,5,6,7,8, \\
9,10,11,12, \\
13,14,15,1 \\
6,\end{array}$ & $\begin{array}{c}4,8,10,11,1 \\
2,13,16\end{array}$ & \multirow{6}{*}{ III } \\
\hline 4 & $\begin{array}{c}4,8,10,11,12,13,15 \\
16\end{array}$ & $\begin{array}{c}3,4,5,6,7,8 \\
9,10,11,12 \\
13,14,15\end{array}$ & $\begin{array}{c}4,8,10,11,1 \\
2,13,15\end{array}$ & \\
\hline 5 & $\begin{array}{c}4,8,10,11,12, \\
13,14,15,16\end{array}$ & $\begin{array}{c}3,4,5,6,7,8 \\
9, \\
10,11,12 \\
13,14\end{array}$ & $\begin{array}{l}4,8,10,11,1 \\
2,13\end{array}$ & \\
\hline 6 & $\begin{array}{c}4,7,9,8,10,11,12,13 \\
, 14,15,16\end{array}$ & $\begin{array}{l}3,4,5,6,7,8, \\
9,11,12,14\end{array}$ & $\begin{array}{c}4,7,9,8,11 \\
12,14\end{array}$ & \\
\hline 7 & $\begin{array}{c}4,5,6,7,9,8,10,11,1 \\
2,13,14,15,16\end{array}$ & 3,5 & 5 & \\
\hline 8 & $\begin{array}{c}2,3,4,5,6,7,9,8,10,1 \\
1,12,13,14,15,16\end{array}$ & 3 & 3 & \\
\hline
\end{tabular}

Table 4.2.3.4: Iteration IV

\begin{tabular}{|c|c|c|c|c|}
\hline $\begin{array}{c}\text { S. } \\
\text { No } \\
\cdot\end{array}$ & $\begin{array}{c}\text { Reachability } \\
\text { set }\end{array}$ & $\begin{array}{c}\text { Antecedent } \\
\text { set }\end{array}$ & $\begin{array}{c}\text { Intersectio } \\
\text { n set }\end{array}$ & $\begin{array}{c}\text { Lev } \\
\text { el }\end{array}$ \\
\hline 4 & $\mathbf{1 5}$ & $\begin{array}{c}3,5,6,7,9,14, \\
15\end{array}$ & 15 & \\
\cline { 1 - 4 } & 14,15 & $3,5,6,7,9,14$ & 14,15 & \\
\hline 6 & $7,9,14,15$ & $3,5,6,7,8,9,14$ & $7,9,14,15$ & \multirow{2}{*}{ IV } \\
\hline 7 & $5,6,7,9,14,15$ & $3,5,6$ & 5,6 & \\
\hline 8 & $3,6,7,9,14,15$ & 3 & 3 & \\
\cline { 1 - 3 }
\end{tabular}

Table 4.2.3.5: Iteration $\mathrm{V}$

\begin{tabular}{|c|c|c|c|c|}
\hline $\begin{array}{l}\text { S. } \\
\text { No }\end{array}$ & $\begin{array}{c}\text { Reachability } \\
\text { set }\end{array}$ & $\begin{array}{c}\text { Antecedent } \\
\text { set }\end{array}$ & $\begin{array}{c}\text { Intersectio } \\
\text { n set }\end{array}$ & $\begin{array}{c}\text { Lev } \\
\text { el }\end{array}$ \\
\hline 4 & 14 & $3,5,6,7,9,14$ & 14 & \multirow[b]{5}{*}{ V } \\
\hline 5 & $7,9,14$ & $3,5,6,7,9,14$ & $7,9,14$ & \\
\hline 6 & $5,6,7,9,14,15$ & $3,5,6$ & 5 & \\
\hline 7 & $6,7,9,14,15$ & $3,5,6$ & 6 & \\
\hline 8 & $3,6,7,9,14,15$ & 3 & 3 & \\
\hline
\end{tabular}

Table 4.2.3.6: Iteration VI

\begin{tabular}{|c|c|c|c|c|}
\hline $\begin{array}{c}\text { S. } \\
\text { No } \\
\cdot\end{array}$ & $\begin{array}{c}\text { Reachability } \\
\text { set }\end{array}$ & $\begin{array}{c}\text { Antecedent } \\
\text { set }\end{array}$ & $\begin{array}{c}\text { Intersectio } \\
\text { n set }\end{array}$ & $\begin{array}{c}\text { Lev } \\
\text { el }\end{array}$ \\
\hline 7 & $\mathbf{5 , 6}$ & $3,5,6$ & 5 & \multirow{2}{*}{ VI } \\
\hline 8 & $3,5,6$ & 3 & 3 & \\
\hline
\end{tabular}

Table 4.2.3.7: Iteration VII

\begin{tabular}{|c|c|c|c|c|}
\hline S.No. & $\begin{array}{c}\text { Reachability } \\
\text { set }\end{array}$ & $\begin{array}{c}\text { Antecedent } \\
\text { set }\end{array}$ & $\begin{array}{c}\text { Intersectio } \\
\text { n set }\end{array}$ & $\begin{array}{c}\text { Lev } \\
\text { el }\end{array}$ \\
\hline 8 & $\mathbf{3}$ & 3 & 3 & VII \\
\hline
\end{tabular}

\section{ACKNOWLEDGMENTS}

Co-author Remica Aggarwal pays her sincere regards to the Asian and Indian weavers particularly struggling women weavers of India who are making a mark in contributing towards the successful running of handloom and carpet weaving small scale enterprises and organizations. She is also thankful to Prof. S.P Singh for disseminating knowledge about ISM methodology.

\section{REFERENCES}

[1] Khatoon, S. 2016. Make in India : A platform to Indian Handloom market, IOSR Journal of business and management (IOSR-IBM). e-ISSN : 2278-487X, 18(9), $36-40$.

[2] M. Rashid Malik, Rekha Prasad, 2015, Indian Carpet Industry after Trade Liberalization : Problems and Prospects , Academic Journal of Economic Studies, Vol. 1, No.3, September 2015, pp. 79-87, ISSN 2393-4913, 
ISSN On-line 2457-5836.

[3] D'Essence Consulting, (2004). The Indian Clusters: An Overview of the Carpet Industry. White Paper Series on Carpet Industry 'Child Labour in the Carpet Industry of India.

[4] Ray, S. (1995). Hand-Knotted Carpet Industry: Myths of 'Export Drive. Economic and Political Weekly, 30 (7/8), 360-362.

[5] Mamidipudi , A. and Bijker, W.E. 2018. Innovation in Indian Handloom Weaving, Technology and Culture 59(3), 509-545 . DOI: 10.1353/tech.2018.0058.

[6] Darbanhosseiniamirkhiz, M., Shabanifar, S., Ismail, W.K.W. (2011). Adoption of Technology in the Hand Woven Carpet Industry: The Case of Alinasab Carpet Company, Australian Journal of Basic and Applied Sciences, 10, 551-559.

[7] Das, P.K.' Shukla, K.P.' and Oryx, F.G., 1992. An Occupational Health Programme for Adults and Children in the Carpet Weaving Industry, Mirzapur, India: A Case Study in the Informal Sector. Sm. Sci. Med., Vol. 35, No. 10, pp-1293-1302.

[8] Juyal, B.N. 1993. Child Labour in Carpet Industry in Mirzapur-Bhadohi. International Labour Organisation, New Delhi.

[9] Saraf, D.N. 1986. Indian Carpets. In silver jubilee special. All India Carpet Manufacturers Association, Bhadohi.

[10] Waziri, A.A. 1986. A Brief History of Carpet Weaving in Bhadohi-Mirzapur Belt. In silver jubilee special. All India Carpets Manufactures Association, Bhadohi.

[11] Singh, S. 1979. Organisation of the Carpet Industry of Bhadohi. Economic and Political Weekly, 14, (21), M69M71.

[12] Venkateswarlu, D., Ramakrishna, RVSS and Moid, M.A. 2006. Child Labour in Carpet Industry in India: Recent Developments. Report of International Labour Rights Fund.

[13] Tyagi, S. 2011. Present scenario of Indian carpet industry vis-a-vis international markets. available at www.wraindia.com/seminar.htm .

[14] Pandey, D.D. 2011..Recession-in-Carpet-Industry. Availableathttp://www.saching.com/Articles/Recessionin-Carpet-Industry-6747.html.

[15] Warfield, J.N. 1974. Developing interconnection matrices in structural modelling. In the proceedings of IEEE Transactions on System, Man, and Cybernetics (SMC), 4 (1), 81-87.

\section{Appendix 1: Persian Carpet Weaving: Types \& Techniques}

By weaving, a process of creating a textile by interlacing threads. Weaving is done on a loom, a frame-like piece of equipment that holds two basic types of threads i.e. a Warp threads which run vertically, attached to the loom frame and held in tension and a Weft threads which run horizontally and are interlaced with warp threads.

Most Persian carpet are made by hand, and their width is determined by the width of the loom on which they are made. Persian carpets may be made of natural fibers like cotton, silk or wool. Persian carpets are famous for a luxurious thick pile or carpet surface that's made by weaving with an important step added. During weaving, many hand-tied knots are added in rows to the warp threads. Then weft threads are interlaced above them and packed down with a comb to hold the knots in place.

$\checkmark \quad$ Knot types include the Persian or Senneh knot, also known as the 'asymmetrical' knot. In this knot, yarn wraps around one warp thread, passes under a neighboring warp, and then goes out to the surface.

$\checkmark \quad$ The Turkish or Ghiorde knot, also known as the 'symmetrical' knot, has yarn wrapped around two warp threads. Each end of the yarn piece is then wrapped behind a warp and brought to the surface. This creates a more durable rug.

$\checkmark \quad$ And then there's the Jufti knot, used in a specific area of Iran, in which a yarn is wrapped around four warp threads.

$\checkmark$ Another type of Persian carpet is a flat weave rug known as a kilim. These rugs don't have knotted pile and they use many special types of weaving techniques that involve skipping a certain number of warp or weft threads to make designs.

\section{Appendix A2 : Case of Mandira Utsav}

- Mandira Utsav is a training-cum-carpet making centre set up by Mojopanda Exim Pvt Ltd, Delhi, in July 2017. This centre is popular amongst women who run their living confidently and with pride knitting and weaving their skills in an extraordinary way.

- This centre is famous for delivering Nepali handknotted, tufted and flat-weave carpets and dhurries . In a day they could weave 6 to 7 inches of high-grade Nepali hand-knotted carpets of 60 to 80 counts.

- Not only this women work at the centre but also manage accounting related tasks such as keeping track of orders received and executed ; maintaining order details as well as registration documents .

- They are also adept at finding and removing mistakes in woven carpets, correcting loose weaving, repairing carpets to the required size as well as binding and giving the finishing touches.

${ }^{1}$ https://study.com/academy/lesson/persian-carpet-weavingtypes-techniques.html.

${ }^{2}$ https://www.thehindubusinessline.com/specials/indiainterior/carpet-weaving-unrolls-economic-opportunitiesfor-these-women/article25852723.ece by Usha Rai [2018]. 